\title{
Polar Constituents of Salvia willeana (Holmboe) Hedge, Growing Wild in Cyprus
}

\author{
Theofilos Mailis and Helen Skaltsa * \\ Department of Pharmacognosy \& Chemistry of Natural Products, School of Pharmacy, National and \\ Kapodistrian University of Athens, Panepistimiopolis, Zografou, 15771 Athens, Greece; skaltsa@pharm.uoa.gr \\ * Correspondence: skaltsa@pharm.uoa.gr; Tel.: +30-21-0727-4593
}

Received: 29 January 2018; Accepted: 1 March 2018; Published: 6 March 2018

\begin{abstract}
Twenty compounds were isolated from the aerial parts of Salvia willeana (Holmboe) Hedge, growing wild in Cyprus. These compounds comprise one new and one known acetophenone, one megastigmane glucoside, five phenolic derivatives, two caffeic acid oligomers, three flavonoids, two lignans, two triterpene acids, one monoterpene glucoside, and two fatty acids. The structures of the isolated compounds were established by means of NMR [(Rotating-frame OverhauserEffect SpectroscopY) $\left({ }^{1} \mathrm{H}^{-1} \mathrm{H}-\mathrm{COSY}\right.$ (COrrelation SpectroscopY), ${ }^{1} \mathrm{H}-{ }^{13} \mathrm{C}-\mathrm{HSQC}$ (Heteronuclear Single Quantum Correlation), HMBC (Heteronuclear Multiple Bond Correlation), NOESY (Nuclear Overhauser Effect SpectroscopY), ROESY (Rotating-frame Overhauser Effect SpectroscopY)] and MS spectral analyses. This is the first report of the natural occurrence of 4-hydroxy-acetophenone 4-O-(3,5-dimethoxy-4-hydroxybenzoyl)- $\beta$-D-glucopyranoside. A chemical review on the non-volatile secondary metabolites has been carried out. Based on the literature data, the analysis revealed that the chemical profile of S. willeana is close to that of S. officinalis L.
\end{abstract}

Keywords: Salvia willeana; 4-hydroxy-acetophenone 4-O-(3,5-dimethoxy-4-hydroxybenzoyl)- $\beta$-D -glucopyranoside; megastigmane glucoside; phenolics; terpenes; 2D NMR; Salvia L: chemical review

\section{Introduction}

The Lamiaceae family consists of more than 250 genera; Salvia $\mathrm{L}$. is the largest genus within this family due to the presence of approximately 900 species. Salvia L. spreads in the warm and temperate regions of both the northern and southern hemispheres, and some species of this genus have been cultivated worldwide for use in folk medicine, in perfumery and cosmetics industries, as well as for culinary purposes, like flavoring and aromatic agents [1,2]. Some of its many interesting biological and pharmacological properties are its antioxidant [3], antimicrobial [3,4], cytotoxic [3,4], anti-HIV [3], and antiplasmodial effects [4], as well as others [3]. It is noteworthy that the name of the genus, Salvia, is derived from the Latin word "salvare", which means "to save", in reference to the curative properties of the plant [5]. The genus has attracted such great interest, that it has become the subject of numerous chemical studies, giving evidence that these plants are a rich source of a wide variety of secondary metabolites, such as polyphenols and terpenoids [1].

Salvia willeana (syn. S. grandiflora subsp. willeana Holmboe and S. grandiflora subsp. albiflora Lindb.) is a low-growing, strongly aromatic suffruticose herb, sometimes carpeting the ground [6]. This species is endemic to Cyprus, where it grows on moist, rocky mountainsides of the Troodos range at 1150-1950 $\mathrm{m}$ altitude and it flowers from May to October [7]. Its extracts possess different pharmacological properties and the plant has been used to halt milk production in nursing mothers, as well as for its antiseptic activity [8]. As S. willeana is locally used in aqueous preparations, the purpose of our study was the investigation of secondary metabolites obtained from the polar extract of its aerial parts. Our previous study of its lipophilic extract, revealed the presence of camphor, 
lupeol, and oleanolic acid, and demonstrated their anti-inflammatory effect [8]. It is of interest to note that there is only one more report regarding the chemical constituents of a polar extract of $S$. willeana, which revealed the presence of the triterpenoids ursolic and oleanolic acids, the diterpenoids carnosic acid and isorosmanol, and the flavonoid salvigenin [9].

\section{Results}

The polar extract (MeOH:H $\mathrm{H}_{2} \mathrm{O}$ 5:1) of $S$. willeana was fractionated by MPLC (medium pressure liquid chromatography), CC (column chromatography), followed by semi-preparative HPLC, preparative TLC, and yielded two triterpenoids, namely ursolic acid (1) and maslinic acid (2), one monoterpene glucoside, $(1 S, 2 R, 4 R)-1,8$-epoxy- $p$-menthan-2-yl-O- $\beta$-D-glucopyranoside (3), one megastigmane glucoside, (6R,9S)-3-oxo- $\alpha$-ionol $\beta$-D-glucopyranoside (4), five phenolic compounds, i.e., hydroxy-tyrosol (5), $p$-anisic acid (6), eleutheroside B (syringin) (7), 1-O- $p$-hydroxybenzoyl- $\beta$-D-apiofuranosyl-( $(1 \rightarrow 6)-\beta$-D-glucopyranoside $(8)$ and eugenylglucoside (9), two acetophenones, 4-O- $\beta$-D-glucopyranosyl acetophenone (picein) (10) and 4-hydroxy-acetophenone 4-O-(3,5-dimethoxy-4-hydroxybenzoyl)- $\beta$-D-glucopyranoside (11), two caffeic acid oligomers, rosmarinic acid (12) and salvianolic acid K (13), three flavonoids, luteolin-7-O- $\beta$-D-glucoside (cynaroside) (14), 6-hydroxyluteolin 7-O- $\beta$-D-glucoside (15) and hesperidin (16), two lignans, syringaresinol-4-O- $\beta$-D-glucopyranoside (17), pinoresinol-4-O- $\beta$-D-glucopyranoside (18), and two fatty acids: linoleic acid (19) and methyl $\alpha$-linolenate (20) (Figure 1).

Compound 11 was obtained as a white amorphous powder. $[a]_{\mathrm{D}}^{20}-4.71^{\circ}($ c $0.25 \mathrm{MeOH})$; $\mathrm{UV}\left(\mathrm{CH}_{3} \mathrm{OH}\right) \lambda_{\max }: 272 \mathrm{~nm}$. The HREIMS of $\mathbf{1 1}$ established its molecular formula as $\mathrm{C}_{23} \mathrm{H}_{26} \mathrm{O}_{11}$ (found 477.1405 [M - H] $]^{-}$, calcd. 478.1449). The ${ }^{1} \mathrm{H}-\mathrm{NMR}$ spectrum (Table 1) showed signals at $7.69(2 \mathrm{H}, \mathrm{d}, J=9.0 \mathrm{~Hz})$ and $7.05(2 \mathrm{H}, \mathrm{d}, J=9.0 \mathrm{~Hz})$, which were indicative of a 1,4-bisubsituted phenyl group. Thus, these proton signals were recognized belonging to the aromatic ring of the acetophenone moiety [10]. In the upfield region of the ${ }^{1} \mathrm{H}-\mathrm{NMR}$ spectrum, a singlet at $\delta_{\mathrm{H}} 2.47(3 \mathrm{H}, \mathrm{s})$ was ascribed to the methyl group $\left(\mathrm{CH}_{3}-8\right)$ attached on the carbonyl group of the acetophenone [10]. Moreover, the presence of a singlet at $\delta_{\mathrm{H}} 7.33$ with an integration of two aromatic protons, indicative of a pair of equivalent methine protons, revealed the occurrence of the syringic acid ester structure in the molecule [11]. In the ${ }^{1} \mathrm{H}$-NMR spectrum, the presence of a $\beta$-D-glucopyranose unit was evident based on a characteristic doublet signal with a coupling constant of $7.9 \mathrm{~Hz}$ at $\delta_{\mathrm{H}} 5.04$, assignable to the anomeric proton of the sugar moiety. Moreover, in the ${ }^{1} \mathrm{H}-{ }^{1} \mathrm{H}-\mathrm{COSY}$ spectrum the correlation peaks between the vicinal protons of the sugar ring were observed. Furthermore, from the ${ }^{13} \mathrm{C}-\mathrm{NMR}$ data (Table 1 ) the carbon signals of the glucose moiety were assigned at $\delta_{\mathrm{C}} 101.7\left(\mathrm{C}-1^{\prime}\right), 77.5\left(\mathrm{C}-3^{\prime}\right), 75.5\left(\mathrm{C}-5^{\prime}\right)$, $74.5\left(\mathrm{C}-2^{\prime}\right), 71.3\left(\mathrm{C}-4^{\prime}\right)$, and $64.8\left(\mathrm{C}-6^{\prime}\right)$, matching the reported data of 1,6-disubstituted- $\beta$-D-glucose [12]. The structural assignment was further confirmed by HSQC experiments, due to the carbon signals at $\delta_{\mathrm{C}}$ 130.1 (C-2/C-6), 116.2 (C-3/C-5), and 26.5 (C-8), indicative of a 4-hydroxy-acetophenone moiety [9,13], while the syringic acid ester was confirmed by the presence of the carbon signals at $\delta_{C} 108.3\left(\mathrm{C}-2^{\prime \prime} / \mathrm{C}-6^{\prime \prime}\right)$, $56.0\left(3^{\prime \prime}, 5^{\prime \prime}-\mathrm{OCH}_{3}\right)[11]$. The existence of the acetophenone moiety was corroborated by the HMBC experiment. This spectrum revealed a long-range cross peak between the carbonyl group at $\delta_{\mathrm{C}}$ 199.1 (C-7) with the equivalent protons $\mathrm{H}-2 / \mathrm{H}-6\left(\delta_{\mathrm{H}} 7.69\right)$, as well as an interaction between the carbonyl group $\left(\delta_{\mathrm{C}} 199.1\right)$ and the methyl group $\mathrm{CH}_{3}-8\left(\delta_{\mathrm{H}} 2.47\right)$. In addition, the linkage of the syringyl moiety with the glucosyl moiety was substantiated by the observation of an HMBC correlation between the carbonyl group at $\delta_{\mathrm{C}} 167.3$ and the $6^{\prime}$-methylene protons at $\delta_{\mathrm{H}} 4.46(\mathrm{dd}, J=11.7,8.0)$. Furthermore, the position of the attachment of the carboxyl group to the quaternary carbon C-1" of the syringic ester was determined by a diagnostic HMBC cross peak between the equivalent methine protons H-2" /H-6" at $\delta_{\mathrm{H}} 7.33$ and the carbonyl carbon C-7" at $\delta_{\mathrm{C}} 167.3$, while a long-range coupling between the methyl protons of the methoxy groups at $\delta_{\mathrm{H}} 3.83$ and the benzylic carbons $\mathrm{C}-3^{\prime \prime} / \mathrm{C}-5^{\prime \prime}$ at $\delta_{\mathrm{C}} 149.0$ was also observed. Moreover, the position of the attachment of glucose to the 4-hydroxy-acetophenone moiety was revealed by a ROESY experiment, which displayed correlations between the anomeric proton $\mathrm{H}-1^{\prime}\left(\delta_{\mathrm{H}} 5.04\right)$ and the equivalent protons $\mathrm{H}-3 / \mathrm{H}-5\left(\delta_{\mathrm{H}} 7.05\right)$ of the 
acetophenone (Figure 2). On the basis of the information above and by comparison with the data for compounds of similar structures $[10,11,13]$ compound $\mathbf{1 1}$ was identified as 4-hydroxy-acetophenone 4-O-(3,5-dimethoxy-4-hydroxybenzoyl)- $\beta$-D-glucopyranoside, which is a new natural product, to the best of our knowledge.

The identification of the known flavonoids luteolin-7-O- $\beta$-D-glucoside (14) [14-16], 6-hydroxyluteolin 7-O- $\beta$-D-glucoside (15) [17], and hesperidin (16) [18-24] was based on UV-VIS and NMR spectroscopic analyses, as well as by comparing their spectroscopic data with those reported in the literature. The structure of the two fatty acids, linoleic acid (19) $[25,26]$ and methyl $\alpha$-linolenate (20) $[26,27]$, has been deduced by the interpretation of NMR and GC-MS data. The ${ }^{1} \mathrm{H}-\mathrm{NMR}$ chemical shifts for compounds $\mathbf{1}$ [28-30], $\mathbf{2}$ [31-33], $\mathbf{3}$ [34,35], $\mathbf{4}$ [35-37], 7 [38-42], 9 [35,43], $\mathbf{1 0}$ [10,35,44], 12 [35,45-48], 17 [49,50], and 18 [51-54] presented in our study are in agreement with the data previously reported in the literature.

However, the NMR data of compounds 5, 6, 8, and 13 are not fully recorded in the literature, therefore, they are presented here below.

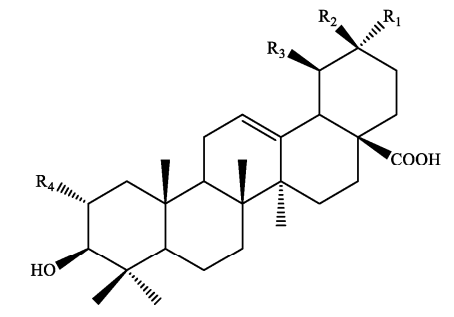

(1) $\mathrm{R}_{1}=\mathrm{R}_{3}=\mathrm{CH}_{3}, \mathrm{R}_{2}=\mathrm{R} 4=\mathrm{H}$ : ursolic acid

(2) $\mathrm{R}_{1}=\mathrm{R}_{2}=\mathrm{CH}_{3}, \mathrm{R}_{3}=\mathrm{H}, \mathrm{R}_{4}=\mathrm{OH}$ : maslinic acid

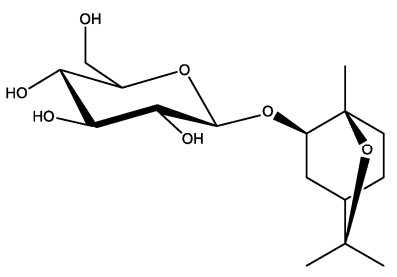

(3) $(1 S, 2 R, 4 R)-1,8$-epoxy$p$-mentha 2-yl-O $\beta$-Dglucopyranoside

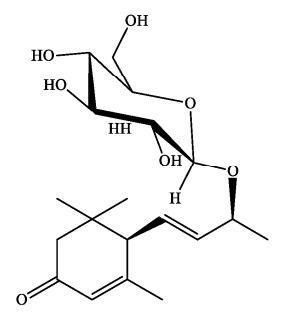

(4) $(6 R, 9 S)-3$-keto- $\alpha$-ionol-9- $\beta$-Dglucopyranoside

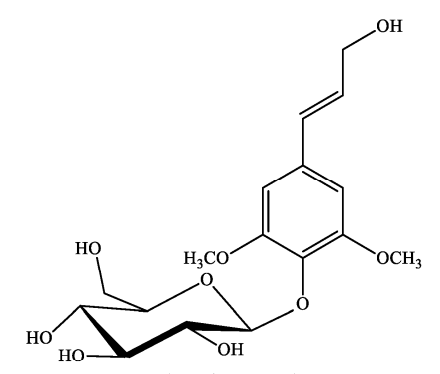

(7) eleutheroside B (syringin)<smiles>COc1ccc(C(=O)O)cc1</smiles>

(6) $p$-anisic acid hydroxytyrosol

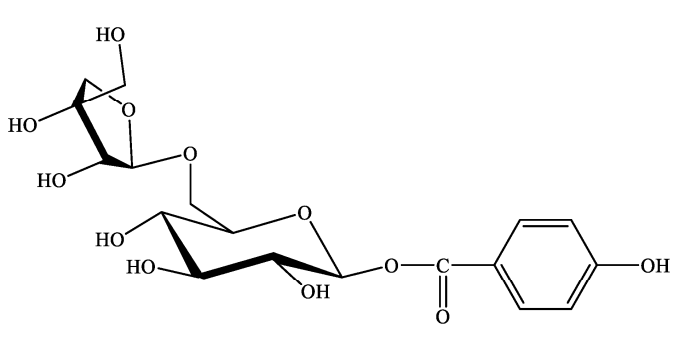

(8) 1-O-p-hydroxybenzoyl- $\beta$-D-apiofuranosyl$(1 \rightarrow 6)-\beta$-D-glucopyranoside

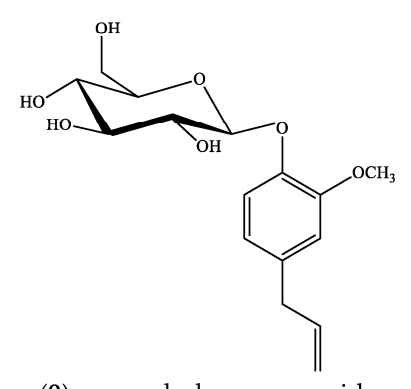

(9) eugenyl-glucopyranoside

Figure 1. Cont. 


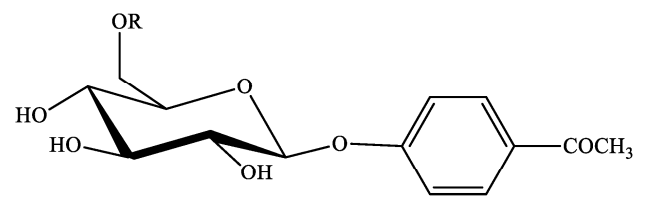

\begin{tabular}{|c|c|c|c|}
\hline (10) & $\mathrm{R}=1$ & & 4-O- $\beta$-D-glucopyranosyl acetophenone (piccin) \\
\hline (11) & $\mathrm{R}=$ & & $\begin{array}{l}\text { 4-hydroxy-acetophen-4-O-(3,5-dimethoxy-4-hydroxybenzoyl)- } \beta \text {-D- } \\
\text { glucopyranoside }\end{array}$ \\
\hline
\end{tabular}

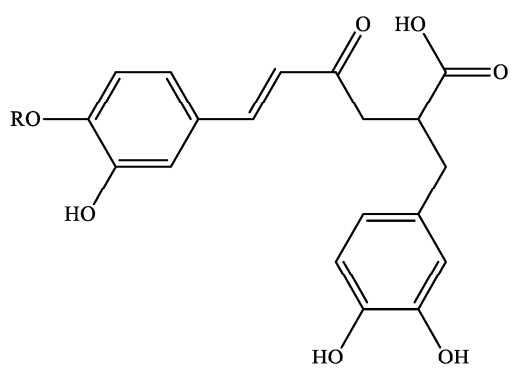

(12)

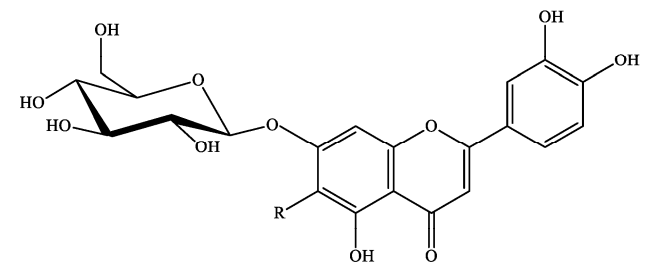

(14): $\mathrm{R}=\mathrm{H}$ luteolin-7-O- $\beta$-D-glucoside (cynaroside) (15): $\mathrm{R}=\mathrm{OH}$ 6-hydroxy-luteolin-7-O- $\beta$-D-glucopyrano side

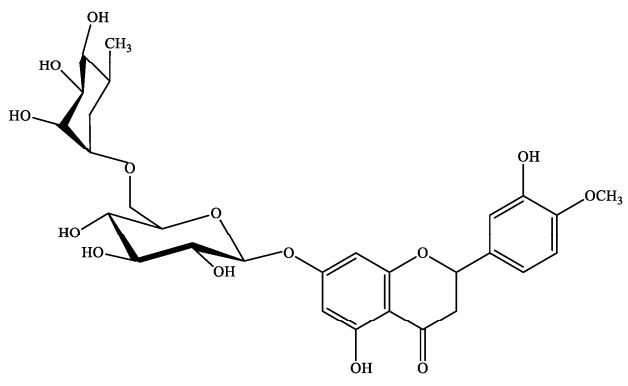

(16) hesperidin

Figure 1. Cont. 


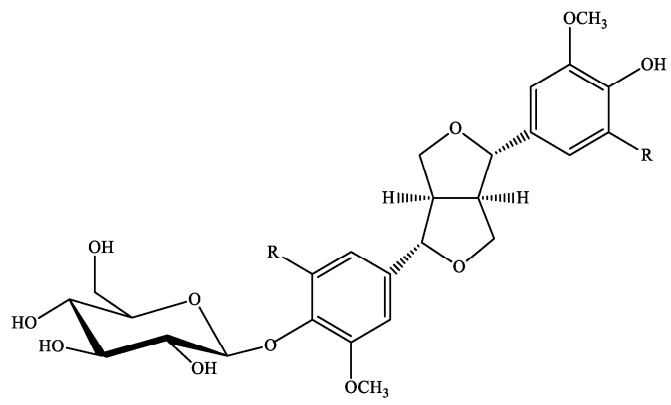

(17) $\mathrm{R}=\mathrm{CH}_{3}$ : syrigaresinol-4-O- $\beta$-D-glucopyranoside

(18) $\mathrm{R}=\mathrm{H}$ : pinoresinol-4-O- $\beta$-D-glucopyranoside

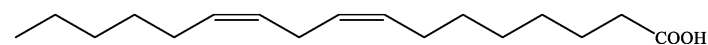

(19) linoleic acid (C18:2)

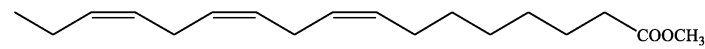

(20) methyl $\alpha$-linolenate (C18:3)

Figure 1. Structures of isolated compounds from Salvia willeana.

Table 1. ${ }^{1} \mathrm{H}-\mathrm{NMR}$ and ${ }^{13} \mathrm{C}-\mathrm{NMR}$ spectrum of $\mathbf{1 1}$.

\begin{tabular}{|c|c|c|c|c|c|}
\hline & $\delta_{\mathrm{C}}$ & $\mathrm{C}$ & $\delta_{\mathbf{H}}$ & $\mathbf{H}$ & $J(\mathrm{~Hz})$ \\
\hline 1 & 131.5 & $\mathrm{C}$ & - & - & - \\
\hline 2 & 130.1 & $\mathrm{CH}$ & 7.69 & 1 & $\mathrm{~d}(J=9.0)$ \\
\hline 3 & 116.2 & $\mathrm{CH}$ & 7.05 & 1 & $\mathrm{~d}(J=9.0)$ \\
\hline 4 & 162.5 & $\mathrm{C}$ & - & - & - \\
\hline 5 & 116.2 & $\mathrm{CH}$ & 7.05 & 1 & $\mathrm{~d}(J=9.0)$ \\
\hline 6 & 130.1 & $\mathrm{CH}$ & 7.69 & 1 & $\mathrm{~d}(J=9.0)$ \\
\hline 7 & 199.1 & C & - & - & - \\
\hline 8 & 26.5 & $\mathrm{CH}_{3}$ & 2.47 & 3 & $\mathrm{~s}$ \\
\hline $1^{\prime}$ & 101.7 & $\mathrm{CH}$ & 5.04 & 1 & $\mathrm{~d}(J=7.8)$ \\
\hline $2^{\prime}$ & 74.5 & $\mathrm{CH}$ & & & \\
\hline $3^{\prime}$ & 77.5 & $\mathrm{CH}$ & 3.53 & 2 & $\mathrm{~m}$ \\
\hline $4^{\prime}$ & 71.3 & $\mathrm{CH}$ & 3.43 & 1 & $\mathrm{~m}$ \\
\hline $5^{\prime}$ & 75.5 & $\mathrm{CH}$ & 3.89 & 1 & $\mathrm{dd}(J=8.0,2.3)$ \\
\hline $6 a^{\prime}$ & & $\mathrm{CH}$ & 4.71 & & $\operatorname{dd}(J=11.7,2.3)$ \\
\hline $6 b^{\prime}$ & 64.8 & $\mathrm{CH}_{2}$ & 4.46 & 2 & $\mathrm{dd}(J=11.7,8.0)$ \\
\hline $1^{\prime \prime}$ & - & $\mathrm{C}$ & - & - & - \\
\hline $2^{\prime \prime}$ & 108.3 & $\mathrm{CH}$ & 7.33 & 1 & $\mathrm{~s}$ \\
\hline $3^{\prime \prime}$ & 149.0 & $\mathrm{C}$ & - & - & - \\
\hline $4^{\prime \prime}$ & 142.3 & $\mathrm{C}$ & - & - & - \\
\hline $5^{\prime \prime}$ & 149.0 & $\mathrm{C}$ & - & - & - \\
\hline $6^{\prime \prime}$ & 108.3 & $\mathrm{CH}$ & 7.33 & 1 & $\mathrm{~s}$ \\
\hline $7^{\prime \prime}$ & 167.3 & $\mathrm{C}$ & - & - & - \\
\hline $\begin{array}{c}3 ", \\
5^{\prime \prime}-\mathrm{OCH}_{3}\end{array}$ & 56.0 & $\mathrm{CH}_{3}$ & 3.83 & 6 & $\mathrm{~s}$ \\
\hline
\end{tabular}




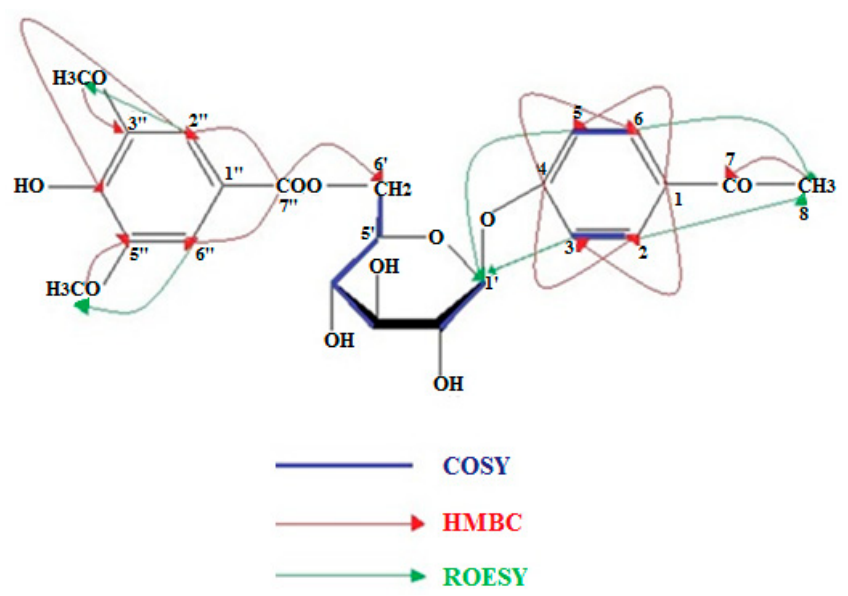

Figure 2. COSY, HMBC, and ROE signals of compund 11.

\section{Discussion}

The genus Salvia L. is characterized by the presence of several different secondary metabolites, mainly phenolic derivatives and terpenoids [3].

In the present study, overall, 20 compounds were isolated from S. willeana polar extracts, i.e., two triterpenoids, namely ursolic acid (1) and maslinic acid (2), one monoterpene glucoside, $(1 S, 2 R, 4 R)-1,8$-epoxy- $p$-menthan-2-yl- $O$ - $\beta$-D-glucopyranoside (3), one megastigmane glucoside, (6R,9S)-3-oxo- $\alpha$-ionol $\beta$-D-glucopyranoside (4), five simple phenolic compounds, i.e., hydroxy-tyrosol (5), $p$-anisic acid (6), eleutheroside B (syringin) (7), 1-O-p-hydroxybenzoyl- $\beta$-D-apiofuranosyl-( $1 \rightarrow 6)$ - $\beta$-D-glucopyranoside (8) and eugenylglucoside (9), two acetophenones, 4-O- $\beta$-D-glucopyranosyl acetophenone (picein) (10) and 4-hydroxy-acetophenone 4-O-(3,5-dimethoxy-4-hydroxybenzoyl)- $\beta$-D-glucopyranoside (11), two caffeic acid oligomers, rosmarinic acid (12) and salvianolic acid K (13), three flavonoids, luteolin-7-O- $\beta$-D-glucoside (cynaroside) (14), 6-hydroxyluteolin 7-O- $\beta$-D-glucoside (15) and hesperidin (16), two lignans, syringaresinol-4-O- $\beta$-D-glucopyranoside (17), pinoresinol-4-O- $\beta$-D-glucopyranoside (18) and two fatty acids: linoleic acid (19) and methyl $\alpha$-linolenate (20).

It is interesting to point out that compounds $7, \mathbf{1 6}, \mathbf{1 7}$, and 18 had not been previously detected in Salvia L. Syringin (7) is reported, here, as a component of the Lamiaceae family for the first time. Moreover, compounds 3, 5, 6, and 8-10 had been previously mentioned only once in the genus, as follows: 3, 9, 10 [35], 6 [55], 8 [10] and 14, 15 [3] in S. officinalis L., 5 in S. digitaloides Diels [56], while compound 4 twice in S. nemorosa and S. officinalis L. [35,37]. As for the two triterpenoids $\mathbf{1}$ and $\mathbf{2}$, these have previously been isolated from the acetone extract of the aerial parts of $S$. willeana [9]. So far, only 134 Salvia species of the over 1000 species suggested have been investigated [3]. Based on our results, concerning the polar secondary metabolites, among these species $S$. willeana showed many similarities to $S$. officinalis L., since most of the isolated simple phenols $(\mathbf{5}, \mathbf{6}, \mathbf{8 - 1 0})$, as well as the flavonoids 14 and 15, are found only in these two species (Supplementary Materials Table S1 and the references herein).

\section{Materials and Methods}

\subsection{Plant Material}

Aerial parts of Salvia willeana (Holmboe) Hedge were collected on Troodos Mountain in Cyprus in April 2004 [8]. A voucher specimen has been deposited in the Agricultural Research Institute Herbarium of Nicosia [no. ARI 3213]. 


\subsection{Equipment and Reagents}

${ }^{1} \mathrm{H},{ }^{13} \mathrm{C}$, and 2D-NMR spectra were recorded in $\mathrm{CDCl}_{3}$ and $\mathrm{CD}_{3} \mathrm{OD}$ on Bruker DRX $400(399.95 \mathrm{MHz}$ for $\left.{ }^{1} \mathrm{H}-\mathrm{NMR}\right)$ and Bruker AC $200\left(200.13 \mathrm{MHz}\right.$ for ${ }^{1} \mathrm{H}-\mathrm{NMR}$ and $50.3 \mathrm{MHz}$ for $\left.{ }^{13} \mathrm{C}-\mathrm{NMR}\right)$ instruments at $295 \mathrm{~K}$ (Bruker BioSpin $\mathrm{GmbH}$, Silberstetten, Germany). Chemical shifts are given in ppm $(\delta)$ and were referenced to the solvent signals at 7.24/3.31 and 77.0/49.5 ppm for ${ }^{1} \mathrm{H}$ and ${ }^{13} \mathrm{C}-\mathrm{NMR}$, respectively. COSY, HSQC, HMBC, NOESY, and ROESY (mixing time $950 \mathrm{~ms}$ ) were performed using standard Bruker microprograms. High-resolution mass spectra were measured on a Q-TOF 6540 UHD (Aligent Technologies, Santa Clara, California, USA). The solvents used were of spectroscopic grade (Merck KGaA, Darmstadt, Germany). UV spectra were recorded on a Shimadzu UV-160A spectrophotometer (Shimadzu; Kyoto, Japan), according to Mabry et al. [57]. Optical rotations were determined using a Perkin-Elmer Polarimeter 341 (Perkin-Elmer, GmbH, Überlingen, Germany). GC-MS (Gas Chromatography-Mass Spectrometry) analyses were performed on a Hewlett-Packard 5973-6890 system (Palo Alto, California) operating in EI mode $(70 \mathrm{eV})$ equipped with a split/splitless injector $\left(220{ }^{\circ} \mathrm{C}\right)$, a split ratio 1/10, using a fused silica HP-5 MS capillary column $(30 \mathrm{~m} \times 0.25 \mathrm{~mm}$ (i.d.), film thickness: $0.25 \mu \mathrm{m})$ with a temperature program for HP-5 MS column from $60{ }^{\circ} \mathrm{C}(5 \mathrm{~min})$ to $280{ }^{\circ} \mathrm{C}$, at a rate of $4{ }^{\circ} \mathrm{C} / \mathrm{min}$ and helium as a carrier gas at a flow rate of $1.0 \mathrm{~mL} / \mathrm{min}$. Preparative HPLC (High-Performance Liquid Chromatography) was performed using a $\mathrm{C}_{18} 25 \mathrm{~cm} \times 10 \mathrm{~mm}$ Kromasil column on a HPLC system (Jasco PU-2080; JASCO, Tokyo, Japan) equipped with an RI detector Shimadzu 10A (Shimadzu, Kyoto, Japan); flow rate: $1.0 \mathrm{~mL} / \mathrm{min}$; concentration of the samples: $3.5-7.0 \mathrm{mg} / \mathrm{mL}$. All solvents used were of HPLC grade (Merck). MPLC (Medium Pressure Liquid Chromatography) was performed using Büchi C-615 and Büchi 688 chromatographic pump; columns: Büchi Borosilikat $3.3,(41.0 \mathrm{~cm} \times 4.0 \mathrm{~cm})$, flow rate: $10 \mathrm{~mL} / \mathrm{min} ;(15.0 \mathrm{~cm} \times 1.5 \mathrm{~cm})$ flow rate: $3 \mathrm{~mL} / \mathrm{min}$; vacuum liquid chromatography (VLC): silica gel 60H (Merck, Art. 7736) [58]. Column chromatography (CC): silica gel (Merck, Art. 9385), silica gel 60 (230-400 mesh ASTM, SDS 2050044) gradient elution with the solvent mixtures indicated in each case; Sephadex LH-20 (Pharmacia Fine Chemicals); cellulose (Avicel, Merck, Art. 2330). Preparative TLC (Thin Layer Chromatography) was performed using pre-coated silica gel 60 plates (Merck, Art. 5721). Fractionation was always monitored by TLC silica gel 60 F-254, (Merck, Art. 5554) with visualization under UV (254 and $365 \mathrm{~nm}$ ) and spraying with vanillin-sulfuric acid reagent (vanillin Merck, Art. No. S26047 841) [59] and Neu's reagent for phenolics [60]. Analytical solvents were obtained from Panreac Quimica SA (Barcelone, Spain, Italy), while deuterated solvents were purchased from Merck, KGaA (Darmstadt, Germany). Di-phosphorus pentoxide was purchased from Chemlab, Belgium.

\subsection{Extraction and Chromatography}

The air-dried powdered aerial parts of $S$. willeana $(0.43 \mathrm{~kg})$ were successively extracted at room temperature with cyclohexane, dichloromethane, $\mathrm{MeOH}$, and $\mathrm{MeOH}: \mathrm{H}_{2} \mathrm{O}$ (5:1) (2 $\mathrm{L}$ of each solvent, twice for $48 \mathrm{~h}$ ) [8]. A portion of the latter extract $(9.0 \mathrm{~g})$ was fractionated on a $\mathrm{RP}_{18}-\mathrm{MPLC}$ $(41.0 \times 4.0 \mathrm{~cm})$ using a $\mathrm{H}_{2} \mathrm{O}$ : $\mathrm{MeOH}$ gradient system $\left(100 \% \mathrm{H}_{2} \mathrm{O} \rightarrow 100 \% \mathrm{MeOH}\right.$; steps of $10 \% \mathrm{MeOH}$; $50 \mathrm{~min}$ each; $50 \% \mathrm{MeOH}$ : 50\% EtOAc $50 \mathrm{~min}$; 100\% EtOAc $50 \mathrm{~min}$ ) to yield twenty three fractions (A-V) of $500 \mathrm{~mL}$ each. Fraction D (598.8 mg; $\left.\mathrm{H}_{2} \mathrm{O}: \mathrm{MeOH} 85: 15\right)$ was similarly purified by $\mathrm{RP}_{18}$-MPLC $(15 \mathrm{~cm} \times 1.5 \mathrm{~cm})$ to obtain three sub-fractions $(1-3)$. The first two sub-fractions were combined together (sub-fraction DA, $364.5 \mathrm{mg}$ ), subjected to a Sephadex LH-20 column and eluted with $100 \%$ methanol to afford 61 fractions combined in 13 groups (DAA-DAM). Group DAJ (4.2 mg) was identified as salvianolic acid K (13). Group DAC (12.9 mg) was subjected to $\mathrm{RP}_{18}$-HPLC (RID; isocratic elution using $\mathrm{MeOH}: \mathrm{CH}_{3} \mathrm{COOH} 5 \%$ 30:70; flow-rate: $1.0 \mathrm{~mL} / \mathrm{min}$ ) and afforded 1-O- $p$-hydroxybenzoyl- $\beta$-D-apiofuranosyl-( $(-6)$ - $\beta$-D-glucopyranoside $(8)(\mathrm{Rt}=14.0 \mathrm{~min}, 0.5 \mathrm{mg})$ and 4-O- $\beta$-D-glucopyranosyl-acetophenone (picein) (10) ( $\mathrm{Rt}=17.2 \mathrm{~min}, 0.7 \mathrm{mg})$. Group DAD (9.3 mg) was purified by prep. TLC on silica gel using $\mathrm{CHCl}_{3}: \mathrm{MeOH}: \mathrm{AcOH}(7: 1.5: 1.5)$ as the eluent and yielded hydroxytyrosol $(6.3 \mathrm{mg})(5)$. Fraction $\mathrm{H}(718.3 \mathrm{mg})$ was fractionated by CC on a Sephadex LH-20 $(25.0 \mathrm{~cm} \times 3.2 \mathrm{~cm})$ using $\mathrm{H}_{2} \mathrm{O}: \mathrm{MeOH}$ (20:80 to 0:100) for gradient elution to afford luteolin 7-O- $\beta$-D-glucoside $(0.7 \mathrm{mg})$ (14). Groups $\mathrm{HC}$ to $\mathrm{HG}$ were combined $\left(\mathrm{HC}^{\prime} ; 193.7 \mathrm{mg}\right.$ ) and purified by 
CC $(12.2 \mathrm{~cm} \times 2.2 \mathrm{~cm})$ over silica gel with cyclohexane: DM:EtOAc:MeOH mixtures of increasing polarity to yield nine groups ( $\left.\mathrm{HC}^{\prime} \mathrm{A}-\mathrm{HC}^{\prime} \mathrm{I}\right)$. Group $\mathrm{HC}^{\prime} \mathrm{C}(14.2 \mathrm{mg}$; eluted with EtOAc:MeOH 97:3 to 94:6) was purified by $\mathrm{RP}_{18}$ - $\mathrm{HPLC}$ (RID; $\mathrm{MeOH} ; \mathrm{H}_{2} \mathrm{O} 40: 60$; flow rate: $1 \mathrm{~mL} / \mathrm{min}$ ) to obtain syringin $(\mathrm{Rt}=56.4 \mathrm{~min}, 0.1 \mathrm{mg})(7),(1 S, 2 R, 4 R)-1,8$-epoxy- $p$-menthan-2-yl-O- $\beta$-D-glucopyranoside $(\mathrm{Rt}=74.2 \mathrm{~min}$, $0.9 \mathrm{mg}$ ) (3), (6R,9S)-3-oxo- $\alpha$-ionol- $\beta$-D-glucopyranoside ( $\mathrm{Rt}=116.5 \mathrm{~min}, 0.5 \mathrm{mg})(4)$, eugenyl-glucoside $(\mathrm{Rt}=130.1 \mathrm{~min}, 0.3 \mathrm{mg})(9)$. Combined groups $\mathrm{HJ}$ to $\mathrm{HL}\left(\mathrm{HJ}^{\prime} ; 48.9 \mathrm{mg}\right.$; eluted with $\mathrm{H}_{2} \mathrm{O}: \mathrm{MeOH}$ 50:50) were subjected to CC over silica gel using cyclohexane: DM:EtOAc:MeOH mixtures of increasing polarity (60 fractions). Purification of fraction $\mathrm{HJ}^{\prime} \mathrm{E}$ (2.1 mg; eluted with EtOAc:MeOH 90:10) was carried out by prep. TLC on silica gel, using $\mathrm{CHCl}_{3}: \mathrm{MeOH}: \mathrm{AcOH}$ (9.0:1.0:0.1) and afforded syringaresinol-4-O- $\beta$-D-glucopyranoside $(0.9 \mathrm{mg})(\mathbf{1 7})$ and pinoresinol-4-O- $\beta$-D-glucopyranoside $(1.2 \mathrm{mg})(\mathbf{1 8})$. Combined groups HM to HP (HM'; $86.7 \mathrm{mg})$ were fractionated by $\mathrm{RP}_{18}$-HPLC ( $\mathrm{MeOH} 58 \%: \mathrm{H}_{2} \mathrm{O} 42 \%$; flow rate: $1 \mathrm{~mL} / \mathrm{min}$ ) and yielded rosmarinic acid ( $\mathrm{Rt}=10.6 \mathrm{~min}, 2.2 \mathrm{mg}$ ) (12) and $p$-anisic acid ( $\mathrm{Rt}=18.9 \mathrm{~min}, 0.1 \mathrm{mg})(6)$, and the sub-fraction $\mathrm{HM}^{\prime} 14 \mathrm{~b}(\mathrm{Rt}=16.7 \mathrm{~min}$, $5.9 \mathrm{mg}$ ), which was further purified by prep. TLC on silica gel with EtOAc:AcOH: $\mathrm{H}_{2} \mathrm{O}$ $(65: 15: 20)$ and led to the isolation of hesperidin $(3.0 \mathrm{mg})(\mathbf{1 6})$ and of 4-hydroxyacetophenone 4-O-(3,5-dimethoxy-4-hydroxybenzoyl)- $\beta$-D-glucopyranoside $(2.8 \mathrm{mg})(\mathbf{1 1})$. Combined groups HT to $\mathrm{HV}\left(\mathrm{HT}^{\prime} ; 176.5 \mathrm{mg}\right)$, subjected to CC on cellulose $(11.0 \mathrm{~cm} \times 3.2 \mathrm{~cm})$ using as eluent $\mathrm{AcOH}: \mathrm{H}_{2} \mathrm{O}$ (30:70) afforded 79 fractions. Fraction $\mathrm{HT}^{\prime} \mathrm{H}(4.8 \mathrm{mg})$ was purified by prep. TLC on silica gel with EtOAc:AcOH: $\mathrm{H}_{2} \mathrm{O}$ (65:15:20) to obtain 6-hydroxyluteolin 7-O- $\beta$-D-glucoside $(0.9 \mathrm{mg})$ (15). The purification of fraction $\mathrm{N}(248.5 \mathrm{mg})$ was performed on silica gel CC $(15.2 \mathrm{~cm} \times 2.0 \mathrm{~cm})$ using mixtures of cyclohexane: DM:EtOAc:MeOH of increasing polarity and afforded methyl $\alpha$-linolenate (C18:3) (1.4 mg) (20) and ursolic acid (4.7 mg) (1). Groups NB' (7.4 mg) and NF (19.6 mg) were subjected to prep. TLC on silica gel with $\mathrm{CHCl}_{3}: \mathrm{MeOH}: \mathrm{AcOH}$ (9.5:0.5:0.05) and yielded linoleic acid (C18:2) $(0.5 \mathrm{mg})(19)$ and maslinic acid (18.3 mg) (2), respectively. All obtained extracts, fractions, and isolated compounds were evaporated to dryness in vacuum under low temperature and then were put in activated desiccators with $\mathrm{P}_{2} \mathrm{O}_{5}$ until their weights had stabilized.

\subsection{NMR Data of $\mathbf{5}, \mathbf{8}$, and $\mathbf{1 3}$}

Compound 5: Yellow amorphous powder; ${ }^{1} \mathrm{H}-\mathrm{NMR}\left(\mathrm{CD}_{3} \mathrm{OD}, 400 \mathrm{MHz}\right): 2.66(2 \mathrm{H}, \mathrm{t}, J=7.3, \mathrm{H}-7)$, $3.66(2 \mathrm{H}, \mathrm{t}, J=7.3, \mathrm{H}-8), 6.52(1 \mathrm{H}, \mathrm{dd}, J=8.0,2.0, \mathrm{H}-6), 6.65(1 \mathrm{H}, \mathrm{d}, J=2.0, \mathrm{H}-2), 6.67(1 \mathrm{H}, \mathrm{d}, J=8.0, \mathrm{H}-5)$.

Compound 6: White amorphous powder; ${ }^{1} \mathrm{H}-\mathrm{NMR}\left(\mathrm{CD}_{3} \mathrm{OD}, 400 \mathrm{MHz}\right): 3.91\left(3 \mathrm{H}, \mathrm{s}, \mathrm{OCH}_{3}\right)$, $6.85(2 \mathrm{H}, \mathrm{d}, J=8.5, \mathrm{H}-3 / \mathrm{H}-5), 7.89(2 \mathrm{H}, \mathrm{d}, J=8.5, \mathrm{H}-2 / \mathrm{H}-6)$.

Compound 8: White amorphous powder; ${ }^{1} \mathrm{H}-\mathrm{NMR}\left(\mathrm{CD}_{3} \mathrm{OD}, 400 \mathrm{MHz}\right): \delta 3.38\left(1 \mathrm{H}, \mathrm{m}, \mathrm{H}-5^{\prime}\right)$, $3.47\left(2 \mathrm{H}, \mathrm{m}, \mathrm{H}-2^{\prime}, \mathrm{H}-3^{\prime}\right), 3.56\left(2 \mathrm{H}, \mathrm{s}, \mathrm{H}-5 \mathrm{a}, \mathrm{b}^{\prime \prime}\right), 3.61\left(1 \mathrm{H}, \mathrm{m}, \mathrm{H}-6 \mathrm{~b}^{\prime}\right), 3.73\left(1 \mathrm{H}, \mathrm{d}, J=9.6, \mathrm{H}-4 \mathrm{~b}^{\prime \prime}\right)$, $3.90\left(1 \mathrm{H}, \mathrm{d}, J=2.2, \mathrm{H}-2^{\prime \prime}\right), 3.96\left(1 \mathrm{H}, \mathrm{d}, J=9.6, \mathrm{H}-4 \mathrm{a}^{\prime \prime}\right), 3.99\left(1 \mathrm{H}, \mathrm{d}, J=11.5, \mathrm{H}-6 \mathrm{a}^{\prime}\right), 4.96(1 \mathrm{H}, \mathrm{d}, J=2.2$, H-1"), $5.64\left(1 \mathrm{H}, \mathrm{d}, J=7.9, \mathrm{H}-1^{\prime}\right), 6.84(2 \mathrm{H}, \mathrm{d}, J=8.7, \mathrm{H}-3, \mathrm{H}-5), 7.96(2 \mathrm{H}, \mathrm{d}, J=8.7, \mathrm{H}-2, \mathrm{H}-6)$.

Compound 13: Yellow amorphous powder; $[a]_{\mathrm{D}}^{20}+0.36^{\circ}(\mathrm{c} 0.350 \mathrm{MeOH}) ;{ }^{1} \mathrm{H}-\mathrm{NMR}\left(\mathrm{CD}_{3} \mathrm{OD}\right.$, $400 \mathrm{MHz}): \delta 2.90\left(1 \mathrm{H}, \mathrm{dd}, J=14.0,9.8, \mathrm{H}^{-} 7^{\prime}\right), 3.07\left(1 \mathrm{H}, \mathrm{dd}, J=14.0,3.8, \mathrm{H}-7^{\prime}\right), 4.24(1 \mathrm{H}, \mathrm{d}, J=6.5$, $\left.\mathrm{H}-8^{\prime \prime}\right), 4.88\left(1 \mathrm{H}, \mathrm{d}, J=6.5, \mathrm{H}-7^{\prime \prime}\right), 5.02\left(1 \mathrm{H}, \mathrm{dd}, J=9.8,3.8, \mathrm{H}-8^{\prime}\right), 6.31(1 \mathrm{H}, \mathrm{d}, J=16.0, \mathrm{H}-8), 6.38(1 \mathrm{H}$, $\mathrm{d}, J=8.0, \mathrm{H}-5), 6.62\left(1 \mathrm{H}, \mathrm{dd}, J=8.0,1.6, \mathrm{H}-6^{\prime}\right), 6.66\left(1 \mathrm{H}, \mathrm{d}, J=8.0, \mathrm{H}-5^{\prime}\right), 6.74\left(1 \mathrm{H}, \mathrm{d}, J=8.0, \mathrm{H}-5^{\prime \prime}\right)$, $6.76\left(1 \mathrm{H}, \mathrm{s}, \mathrm{H}-2^{\prime}\right), 6.82(1 \mathrm{H}, \mathrm{dd}, J=8.0,2.0, \mathrm{H}-6), 6.84\left(1 \mathrm{H}, \mathrm{dd}, J=8.0,2.0, \mathrm{H}-6^{\prime \prime}\right), 6.98(1 \mathrm{H}, \mathrm{d}, J=2.0$, H-2"), $7.01(1 \mathrm{H}, \mathrm{d}, J=2.0, \mathrm{H}-2), 7.47(1 \mathrm{H}, \mathrm{d}, J=16.0, \mathrm{H}-7)$.

Supplementary Materials: The following are available online at www.mdpi.com/2223-7747/7/1/18/s1. Table S1: Non-volatile secondary metabolites of Salvia L.

Acknowledgments: The authors thank J. Heilmann (University of Regensburg, Pharmaceutical Biology), as well as J. Kiermaier for recording the MS spectra (all central analytics of NWF IV, University of Regensburg, Germany).

Author Contributions: T.M. contributed to the writing and carried out all chemical analyses. H.S. conceived and designed the experiments, contributed to the writing, and also supervised the chemical analyses.

Conflicts of Interest: The authors declare no conflict of interest. 


\section{References}

1. Lu, Y.; Foo, L.Y. Polyphenolics of Salvia-A review. Phytochemistry 2002, 59, 117-140. [CrossRef]

2. Walker, J.B.; Systma, K.J. Staminal Evolution in the genus Salvia (Lamiaceae). Ann. Bot. 2007, 100, $375-391$. [CrossRef] [PubMed]

3. Wu, Y.-B.; Ni, Z.-Y.; Shi, Q.-W.; Dong, M.; Kiyota, H.; Gu, Y.-C.; Cong, B. Constituents from Salvia Species and Their Biological Activities. Chem. Rev. 2012, 112, 5967-6026. [CrossRef] [PubMed]

4. Kamatou, G.P.P.; Makunga, N.P.; Ramogola, W.P.N.; Viljoena, A.M. South African Salvia species: A review of biological activities and phytochemistry. J. Ethnopharmacol. 2008, 119, 664-672. [CrossRef] [PubMed]

5. Grieve, M. A Modern Herbal; Savas Publishing: El Dorado Hills, CA, USA, 1984; ISBN 139780486227986.

6. Dereboylu, A.; Şengonca, N.; Güvensen, A.; Gücel, S. Anatomical and palynological characteristics of Salvia willeana (Holmboe) Hedge and Salvia veneris Hedge endemic to Cyprus. Afr. J. Biotechnol. 2010, 9, 2076-2088.

7. Bellomaria, B.; Arnold, N.; Valentini, G. Contribution to the study of the essential oils from three species of Salvia growing wild in the eastern Mediterranean region. J. Essent. Oil Res. 1992, 4, 607-614. [CrossRef]

8. Vonaparti, A.; Karioti, A.; Recio, M.; Máñez, S.; Ríos, J.; Skaltsa, E.; Giner, R. Effects of terpenoids from Salvia willeana in delayed-type hypersensitivity, human lymphocyte proliferation and cytokine production. Nat. Prod. Commun. 2008, 3, 1953-1958.

9. De la Torre, M.; Bruno, M.; Savona, F.; Rodriquez, B.; Apostolides Arnold, N. Terpenoids from Salvia willeana and S. virgata. Phytochemistry 1990, 29, 668-670. [CrossRef]

10. Wang, M.; Kikuzaki, H.; Lin, C.; Kahyaoglu, A.; Huang, M.; Nakatani, N.; Ho, C. Acetophenone Glycosides from Thyme (Thymus vulgaris L.). J. Agric. Food Chem. 1999, 47, 1911-1914. [CrossRef] [PubMed]

11. Machida, K.; Yogiashi, Y.; Matsuda, S.; Suzuki, A.; Kikuchi, M. A new phenolic glycoside syringate from the bark of Juglans mandshurica MAXIM. var. sieboldiana MAKINO. J. Nat. Med. 2009, 63, 220-222. [CrossRef] [PubMed]

12. Wang, M.; Kikuzaki, H.; Zhu, N.; Sang, S.; Nakatani, N.; Ho, C.-T. Isolation and structural elucidation of two new glycosides from sage (Salvia officinalis L.). J. Agric. Food Chem. 2000, 48, 235-238. [CrossRef] [PubMed]

13. Olennikov, D.; Chekhirova, G.V. 6"-Galloylpicein and other phenolic compounds from Arctostaphylos uva-ursi. Chem. Nat. Compd. 2013, 49, 1-7. [CrossRef]

14. Özgen, U.; Sevindik, H.; Kazaz, C.; Yigit, D.; Kandemir, A.; Secen, H.; Calis, I. A new sulfated a-Ionone glycoside from Sonchus erzincanicus Matthews. Molecules 2010, 15, 2593-2599. [CrossRef] [PubMed]

15. Benayache, F.; Boureghda, A.; Ameddah, S.; Marchioni, E.; Benayache, S. Flavonoids from Thymus numidicus Poiret. Pharm. Lett. 2014, 6, 50-54.

16. Rashed, K.; Ćirić, A.; Glamočlija, J.; Calhelha, R. Antimicrobial and cytotoxic activities of Alnus rugosa L. aerial parts and identification of the bioactive components. Ind. Crops Prod. 2014, 59, 189-196. [CrossRef]

17. Lu, Y.; Foo, L.Y. Flavonoid and phenolic glycosides from Salvia officinalis. Phytochemistry 2000, 55, 263-267. [CrossRef]

18. Ikan, R. Natural Products. A Laboratory Guide, 2nd ed.; Academic Press Inc.: Cambridge, MA, USA, 1991; pp. 9-11.

19. Carvalho, M.G.; Costa, P.M.; Santos Abreu, H. Flavones from Vernonia diffusa. J. Braz. Chem. Soc. 1999, 10, 163-166. [CrossRef]

20. Marder, M.; Viola, H.; Wasowski, C.; Fernández, S.; Medina, J.; Paladini, A. 6-Methylapigenin and hesperidin, new valeriana flavonoids with activity on the CNS. Pharmacol. Biochem. Behav. 2003, 75, 537-545. [CrossRef]

21. Aghel, N.; Ramezani, Z.; Beiranvard, S. Hesperidin from Citrus sinensis Cultivated in Dezful, Iran. Pak. J. Biol. Sci. 2008, 11, 2451-2453. [CrossRef] [PubMed]

22. Nizamutdinova, I.T.; Jeong, J.J.; Xu, G.H.; Lee, S.; Kang, S.S.; Kim, Y.S.; Chang, K.C.; Kim, H.J. Hesperidin, hesperidin methyl chalone and phellopterin from Poncirus trifoliata (Rutaceae) differentially regulate the expression of adhesion molecules in tumor necrosis factor- $\alpha$-stimulated human umbilical vein endothelial cells. Int. Immunopharmacol. 2008, 8, 670-678. [CrossRef] [PubMed]

23. Maltese, F.; Erkelens, C.; Kooy, F.; Choi, Y.; Verpoorte, R. Identification of natural epimeric flavanone glycosides by NMR spectroscopy. Food Chem. 2009, 116, 575-579. [CrossRef]

24. Han, S.; Mok, S.; Kim, H.; Lee, J.; Lee, D.; Lee, S.Y.; Kim, J.; Kim, S.; Lee, S. Determination of hesperidin in mixed tea by HPLC. J Agric. Sci. 2011, 38, 295-299.

25. Lee, C.K.; Chang, M.H. The chemical constituents from the heartwood of Eucalyptus citriodora. J Chin. Chem. Soc. 2000, 47, 555-560. [CrossRef] 
26. Hatzakis, E.; Agiomyrgianaki, A.; Kostidis, S.; Dais, P. High-resolution NMR spectroscopy, an alternative fast tool for Qualitative and Quantitative Analysis of Diacylglycerol (DAG) oil. J. Am. Oil Chem. Soc. 2011, 88, 1695-1708. [CrossRef]

27. Horst, M.; Urbin, S.; Burton, R.; MacMillan, C. Using proton nuclear magnetic resonance as a rapid response research tool for methyl ester characterization in biodiesel. Lipid Technol. 2009, 21, 1-3. [CrossRef]

28. Güvenalp, Z.; Özbek, H.; Kuruüzüm-Uz, A.; Kazaz, C.; Demirezer, Ö.L. Secondary metabolites from Nepeta heliotropifolia. Turk. J. Chem. 2009, 33, 667-675.

29. Ragasa, Y.; Ng, V.A.S.; Ebajo, V.D.; Fortin, D.R.; De Los Reyes, M.M.; Shen, C.-C. Triterpenes from Shorea negrosensis. Pharm. Lett. 2014, 6, 453-458.

30. Raza, R.; Ilyas, Z.; Ali, S.; Muhammad, N.M.; Muhammad, Y.; Khokhar, M.Y.; Iqbal, J. Identification of Highly Potent and Selective $\alpha$-Glucosidase Inhibitors with Antiglycation Potential, Isolated from Rhododendron arboreum. Rec. Nat. Prod. 2015, 9, 262-266.

31. Tanaka, J.C.A.; Vidotti, G.J.; da Silva, C.C. A New Tormentic Acid Derivative from Luehea divaricata Mart. (Tiliaceae). J. Braz. Chem. Soc. 2003, 14, 475-478. [CrossRef]

32. Rungsimakan, S. Phytochemical and Biological Activity Studies on Salvia viridis L. Ph.D. Thesis, Department of Pharmacy and Pharmacology, University of Bath, Bath, UK, 2011.

33. Woo, W.K.; Han, Y.J.; Un Choi, S.; Ki, H.; Kim, K.H.; Lee, K.R. Triterpenes from Perilla frutescens var. acuta and Their Cytotoxic Activity. Nat. Prod. Sci. 2014, 20, 71-75.

34. Manns, D. Linalool and cineole type glucosides from Cunila spicata. Phytochemistry 1995, 39, 1115-1118. [CrossRef]

35. Wang, M.; Shao, Y.; Huang, T.; Wei, G.; Ho, C. Isolation and structural elucidation of aroma constituents bound as glucosides from Sage (Salvia officinalis). J. Agric. Food Chem. 1998, 46, 2509-2511. [CrossRef]

36. Pabst, A.; Barron, D.; Sémon, E.; Schreier, P. Two diastereomeric 3-oxo- $\alpha$-ionol $\beta$-D-glucosides from Raspberry fruit. Phytochemistry 1992, 31, 1649-1652. [CrossRef]

37. Takeda, Y.; Zhang, H.; Matsumoto, T.; Otsuka, H.; Oosio, Y.; Honda, G.; Tabata, M.; Fujita, T.; Su, H.; Sezik, E.; et al. Megastigmane glycosides from Salvia nemorosa. Phytochemistry 1997, 44, 117-120. [CrossRef]

38. Sugiyama, M.; Nagayama, E.; Kikuchi, M. Lignan and Phenylpropanoid glycosides from Osmanthus asiaticus. Phytochemistry 1993, 33, 1215-1219. [CrossRef]

39. Kim, M.; Moon, H.; Lee, D.; Woo, E. A new lignan glycoside from the Stem bark of Styrax japonica S. et Z. Arch. Pharm. Res. 2007, 30, 425-430. [CrossRef] [PubMed]

40. Qin, Y.; Yin, C.; Cheng, Z. A new tetrahydrofuran lignan diglycoside from Viola tianshanica Maxim. Molecules 2013, 18, 13636-13644. [CrossRef] [PubMed]

41. Lee, J.; Lee, M.; Pae, S.; Oh, K.; Jung, C.; Baek, I.; Lee, S. Analysis of yield of eleutherosides B and E in Acanthopanax divaricatus and A. koreanum Grown with varying cultivation methods. Sci. World J. 2014. [CrossRef]

42. Lall, N.; Kishore, N.; Binneman, B.; Twilley, D.; Venter, M.; Plessis-Stoman, D.; Boukes, G.; Hussein, A. Cytotoxicity of Syringin and 4-methoxycinnamyl alcohol isolated from Foeniculum vulgare on selected human cell lines. Nat. Prod. Res. 2015, 1752-1756. [CrossRef] [PubMed]

43. Mulkens, A.; Kapetanidis, I. Eugenylglucoside, a new natural phenylpropanoid heteroside from Melissa officinalis. J. Nat. Prod. 1988, 51, 496-498. [CrossRef] [PubMed]

44. Ushiyama, M.; Furuya, T. Glycosylation of phenolic compounds by root culture of Panax ginseng. Phytochemistry 1989, 28, 3009-3013. [CrossRef]

45. Lu, Y.; Foo, L.Y. Rosmarinic acid derivatives from Salvia officinalis. Phytochemistry 1999, 51, 91-94. [CrossRef]

46. Woo, E.; Piao, M. Antioxidative Constituents from Lycopus lucidus. Arch. Pharm. Res. 2004, 27, $173-176$. [CrossRef] [PubMed]

47. Özgen, U.; Mavi, A.; Terzi, Z.; Kazaz, C.; Aşçi, A.; Kaya, Y.; Seçen, H. Relationship between chemical structure and antioxidant activity of luteolin and its glyxosides isolated from Thymus sipyleus subsp. sipyleus var. sipyleus. Rec. Nat. Prod. 2011, 5, 12-21.

48. Dapkevicius, A.; Beek, T.; Lelyveld, G.; Veldhuizen, A.; Groot, A.; Linssen, J.; Venskutonis, R. Isolation and structure Elucidation of radican scavengers from Thymus vulgaris Leaves. J. Nat. Prod. 2002, 65, 892-896. [CrossRef] [PubMed]

49. Shahat, A.A.; Abdel-Azim, N.S.; Pieters, L.; Vlietinck, A.J. Isolation and NMR spectra of syringaresinol- $\beta$-D-glucoside from Cressa cretica. Fitoterapia 2004, 75, 771-773. [CrossRef] [PubMed] 
50. Park, H.B.; Lee, K.H.; Kim, K.H.; Lee, I.K.; Noh, H.J.; Choi, S.U.; Lee, K.R. Lignans from the roots of Berberis amurensis. Nat. Prod. Sci. 2009, 15, 17-21.

51. Klimek, B.; Tokar, M. Biologically active compounds from the flowers of Forsythia suspensa Vahl. Acta Pol. Pharm. 1998, 55, 499-504.

52. Ouyang, M.; Shung, Y.; Zhang, Z.K.; Kuo, Y.H. Inhibitory Activity against Tobacco Mosaic Virus (TMV) Replication of Pinoresinol and Syringaresinol Lignans and Their Glycosides from the Root of Rhus javanica var. roxburghiana. J. Agric. Food Chem. 2007, 55, 6460-6465. [CrossRef] [PubMed]

53. Chiou, W.; Shen, C.; Lin, L. Anti-inflammatory principles from Balanopora laxiflora. J. Food Drug Anal. 2011, 19, 502-508.

54. Kim, A.R.; Ko, H.J.; Chowdhury, M.A.; Chang, Y.; Woo, E. Chemical constituents of the aerial parts of Artemisia selengensis and their IL-6 inhibitory activity. Arch. Pharm. Res. 2015, 38, 1059-1065. [CrossRef] [PubMed]

55. Jerković, I.; Mastelić, J.; Marijanović, Z. A variety of volatile compounds as Markers in Unifloral Honey from Dalmatian Sage (Salvia officinalis L.). Chem. Biodivers. 2006, 3, 1307-1316. [CrossRef] [PubMed]

56. Wu, S.; Chan, Y. Five new iridoids from roots of Salvia digitaloides. Molecules 2014, 19, 15521-15534. [CrossRef] [PubMed]

57. Mabry, T.G.; Markham, K.R.; Thomas, M.B. The Systematic Identification of Flavonoids; Springer: New York, NY, USA, 1970.

58. Coll, J.C.; Bowden, B.F. The application of Vacuum Liquid Chromatography to the separation of terpene mixtures. J. Nat. Prod. 1986, 49, 934-936. [CrossRef]

59. Stahl, E. Thin-Layer Chromatography, a Laboratory Handbook, 2nd ed.; Springer: Berlin/Heideiberg, Germany, 1969.

60. Neu, R. Chelate von Diarylborsäuren mit aliphatischen Oxyalkylaminen als Reagenzien für den Nachweis von Oxyphenyl-benzo- $\gamma$-pyronen. Naturwissenschaften 1957, 44, 181-183. [CrossRef]

(C) 2018 by the authors. Licensee MDPI, Basel, Switzerland. This article is an open access article distributed under the terms and conditions of the Creative Commons Attribution (CC BY) license (http:/ / creativecommons.org/licenses/by/4.0/). 